\title{
Evaluación de la calidad físico-química de las aguas subterráneas y superficiales de la zona minera de Santo Domingo - Chontales.
}

\section{Lic. Maybis López Hernández}

Especialista en Análisis de laboratorio

UNAN-Managua, Laboratorio de Biotecnología

https://orcid.org/0000-0002-4523-3129

mlopezh@unan.edu.ni

\section{Ing. Anielka Dávila López}

Técnico de investigación

UNAN-Managua, Laboratorio de Biotecnología https://orcid.org/0000-0001-5033-4955

adavila@unan.edu.ni

\section{Dra. Martha Lacayo Romero \\ Docente titular \\ UNAN-Managua, Laboratorio de Biotecnología \\ https://orcid.org/0000-0002-6918-7796 \\ mlacayor@unan.edu.ni}

Fecha de recepción: 17 de marzo, 2020 / Fecha de aceptación: 07 de julio, 2020

https://doi.org/10.5377/torreon.v9i26.10263

Palabras clave: índice de calidad de agua, usos del agua, aguas subterráneas y superficiales, minería

\section{RESUMEN}

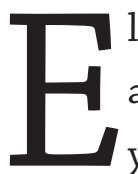

1 objetivo de este estudio fue estimar la calidad físico-química de cinco fuentes de agua, Peñas Blancas y Túnel Azul (subterráneas), El Sardinillo, plantel La Estrella y El Salto (superficiales) de la zona minera de Santo Domingo, Chontales. Se realizaron cinco muestreos a lo largo del río Artiguas en época lluviosa comprendidos desde septiembre hasta diciembre del 2013. Los valores obtenidos se compararon con normas o directrices nacionales e internacionales como CAPRE (Norma de Calidad del Agua para Consumo Humano), OMS (Organización Mundial para la Salud), CCME (Consejo Canadiense de Ministros del Medio Ambiente), FAO (Organización de Agricultura y Alimento) y USEPA (Agencia de 
Protección Ambiental de Estados Unidos) según los usos del agua, consumo humano, protección para la vida acuática y uso agrícola. Además, se calculó un valor numérico entre 0 a 100 de calidad del agua para cada sitio mediante el Software CWQI 1.0. Los índices de calidad para las aguas subterráneas se clasifican como excelentes para consumo humano y uso agrícola, siendo la calidad del agua del Túnel Azul ligeramente superior a la del pozo Peñas Blancas. El Sardinillo sitio de referencia de las aguas superficiales presenta una calidad desde regular a buena para consumo humano, pobre a buena para la protección de la vida acuática y excelente para uso agrícola considerados en las normas o criterios establecidos. El plantel, La Estrella y las del Salto presentan calidades semejantes para consumo humano y protección de la vida acuática desde pobre a marginal, así mismo regular a excelente para el uso agrícola según las consideraciones de los valores guías que se establecen en las normas. La calidad físico-química estimada para las aguas superficiales indica que no pueden ser utilizadas para consumo humano, no obstante, si pueden ser útiles para agricultura.

\section{INTRODUCCIÓN}

El agua es un recurso esencial para la vida y debe ser un suministro adecuado, seguro y accesible que pueda resultar en beneficios tangibles para la salud (OMS, 2019). La calidad del agua puede cambiar debido a condiciones climáticas, eventos naturales del entorno ambiental y la influencia de las actividades antropogénicas, tales como domésticas, industriales, ganadería, minería, entre otras, que hacen que un recurso tan importante no sea apta para sus diferentes usos, siendo la minería una de las actividades que provoca mayor contaminación (WHO, 2011). La calidad del agua, según su uso, tiene un impacto importante en la salud, ya que el agua de mala calidad causa brotes de enfermedades agudas o crónicas tales como, cólera, diarrea, infecciones intestinales, parasitosis, tifoidea, hepatitis, alergias, enfermedades de la piel, cáncer, etc. (OMS, 2006).

Nicaragua cuenta con 28 distritos mineros, que en su mayoría se encuentran concentrados en la parte norte del país en la frontera con Honduras. Entre los principales recursos mineros se encuentran el oro, la plata, el cobre y el plomo, también yacimientos de molibdeno, zinc, hierro, tungsteno y antimonio (Nolasco, S., CEICOM, 2011). En Santo Domingo, Chontales, gran parte de la actividad minera se realiza en las riberas del Río Artiguas, provocando una importante pérdida de recursos naturales como reservas de agua, tierras cultivables, erosión del suelo, contaminación y pérdida de bosques (Flores, M., E., 2009). En la actividad minera artesanal y a pequeña escala se utiliza el mercurio $(\mathrm{Hg})$ o mezclado con cianuro $(\mathrm{Hg} / \mathrm{CN})$ durante el proceso de amalgamación en instalaciones conocidas como rastras. El residuo generado en la molienda se deposita en planteles o cerca a los sitios de extracción donde se expone a la meteorización que 
facilita el transporte de este material a fuentes de aguas u otros receptores ecológicos. (Picado F., Mendoza A. , Cuadra S., Barmen G., Jakobsson K., and G, 2010).

El objetivo de esta investigación fue evaluar la calidad físico-química de las aguas subterráneas y superficiales de la zona minera de Santo Domingo - Chontales utilizando estándares, directrices o criterios recomendados por agencias nacionales e internacionales que establecen guías o valores máximos permisibles para los diferentes usos del agua. Así mismo, se utilizó el software WQI 1.0 herramienta útil para describir el estado de la columna de agua, los sedimentos y la vida acuática (CCME, 2001). Los resultados obtenidos podrán ser utilizados en el establecimiento de monitoreos y planes de manejos de los recursos hídricos.

\section{MATERIALES Y MÉTODOS}

Este estudio fue realizado en la zona minera de Santo Domingo, Chontales, ubicado al nor-occidente del territorio del municipio del mismo nombre. La microcuenca en estudio es la del río Artiguas, afluente al río Sucio Sur, que descarga sus aguas al Río Siquia, y junto con los ríos Mico y Rama desembocan al río Escondido. Finalmente, la cuenca termina en el Océano Atlántico (Espinoza, T., \& Espinoza Benavides L., 2005). Se colectaron las muestras de agua subterránea y en agua superficial en cinco fechas de muestreo, en época lluviosa, distribuidas desde septiembre a diciembre del año 2013. (Tabla 1)

Tabla 1. Sitios de muestreo en el río Artiguas

\begin{tabular}{|l|c|c|}
\hline \multirow{2}{*}{\multicolumn{1}{|c|}{ Sitio }} & \multicolumn{2}{c|}{ Coordenadas } \\
\cline { 2 - 3 } & N & E \\
\hline ASub - Túnel Azul & 1355246 & 708855 \\
\hline PP - Peñas Blancas & 1357193 & 709637 \\
\hline AS-El Sardinillo & 1357522 & 709570 \\
\hline AS-Plantel La Estrella & 1357267 & 709535 \\
\hline AS-El Salto & 1355974 & 708675 \\
\hline
\end{tabular}

El primer punto fue El Túnel Azul, un manantial donde los habitantes de Santo Domingo se abastecen para consumo. El segundo punto fue un pozo perforado conocido como Peñas Blancas ubicado en la finca llamada el Jordán con el fin de reemplazar al Túnel Azul como fuente de abastecimiento y aprovechar el potencial minero que posee la zona donde está ubicado el manantial. 
Las muestras de aguas superficiales fueron colectadas en tres puntos a lo largo del río Artiguas. El primer punto fue el sitio de referencia El Sardinillo, aguas arriba del área de impacto de la actividad minera. El segundo sitio fue El plantel La Estrella, donde se depositaban residuos mineros y que está cercano al río. El tercer punto fue El Salto, aguas abajo, situado en los límites de la ciudad donde se ha recibido efluentes y escorrentía de los planteles, además de las aguas residuales de la ciudad.

Las muestras fueron colectadas de forma puntual en cada uno de los sitios. Para los parámetros físico-químicos $\mathrm{pH}$, conductividad, temperatura, alcalinidad total, cloruros, sólidos sedimentables, sólidos suspendidos totales y sulfatos las muestras fueron almacenadas bajo refrigeración a $4^{\circ} \mathrm{C}$ hasta su análisis. Para el análisis de metales totales recuperables y metales disueltos las muestras fueron preservadas con $\mathrm{HNO}_{3}$ concentrado, sin embargo, para el análisis de metales disueltos las muestras fueron filtradas al momento de su colecta. La metodología y la técnica empleada se presentan en la tabla 2 para cada uno de los parámetros.

Tabla 2. Parámetros de interés para el estudio y método implementado para el análisis

\begin{tabular}{|l|l|}
\hline Parámetros evaluados & $\begin{array}{l}\text { Métodos SMWW usados (American Public Health Associa- } \\
\text { tion, American Water Works Association, \& Water Enviroment } \\
\text { Federation, 2012) }\end{array}$ \\
\hline $\begin{array}{l}\text { Metales y metaloides: } \\
\text { Al, Sb, As, Ba, Be, B, Ca, } \\
\mathbf{C u}, \mathbf{C r}, \mathbf{F e}, \mathbf{L i}, \mathbf{M g}, \mathbf{M n}, \\
\mathbf{H g}, \mathbf{N i}, \mathbf{P b}, \mathbf{K}, \mathbf{S e}, \mathbf{N a}, \mathbf{T 1}, \\
\mathbf{V}, \mathbf{Z n}\end{array}$ & $\begin{array}{l}\text { 3030 F. Digestión con ácido nítrico y clorhídrico método } 3120 \\
\text { Inductivamente }\end{array}$ \\
\hline $\begin{array}{l}\text { Alcalinidad total } \\
\text { Cloruros }\end{array}$ & 2320B. Método titrimétrico \\
\hline $\begin{array}{l}\text { Conductividad y } \\
\text { Temperatura }\end{array}$ & 4500-Cl B. Método argentométrico \\
\hline pH & 2510B. Método de laboratorio \\
\hline Sólidos sedimentables & 2540 F. Análisis volumétrico en Cono Imhoff \\
\hline $\begin{array}{l}\text { Sólidos Suspendidos } \\
\text { Totales }\end{array}$ & 2540.D. Método gravimétrico. Secados a 103-105 ${ }^{\circ} \mathrm{C}$ \\
\hline Sulfatos & $4500-S O 4$ E. Método turbidimétrico \\
\hline
\end{tabular}




\section{Directrices nacionales e internacionales que rigen la calidad del agua}

Se estimó la calidad del agua comparando los resultados con estándares, directrices o criterios recomendados por agencias nacionales e internacionales como: Norma de Calidad del Agua para Consumo Humano (CAPRE, 1994), Guías establecidas por Organización Mundial para la Salud (OMS, 2006), Consejo Canadiense de Ministros del Medio Ambiente (CCME, 2019), guías establecidas por la Organización de Agricultura y Alimento (Ayers R. S., Westcot D. W., \& FAO, 1985) y las normas descritas por la Agencia de Protección Ambiental de Estados Unidos (USEPA, 2017). Estas agencias establecen valores para los distintos usos del agua, consumo humano, protección de la vida acuática (aguda y crónica) y uso agrícola (irrigación y ganadería).

En la tabla 3 y 4 se describen los rangos máximos permisibles establecidos por normas y directrices nacionales e internacionales para la calidad del agua según su uso. Cabe señalar que los valores que se establecen en las tablas solamente son para los parámetros que no cumplieron con lo establecido por las normas.

Tabla 3. Normas, directrices, estándares o valores guías para consumo humano

\begin{tabular}{|l|l|l|l|l|}
\hline \multicolumn{1}{|c|}{ Parámetro } & \multicolumn{1}{c|}{ CAPRE } & \multicolumn{1}{|c|}{ OMS } & \multicolumn{1}{|c|}{ USEPA } & \multicolumn{1}{c|}{ CCME } \\
\hline & $1993 / 1994$ & 2011 & 2015 & 2008 \\
\hline $\mathrm{pH}$ & $6,5-8,5$ & $\mathrm{NE}$ & $6,5-8,5$ & $6,5-8,5$ \\
\hline Aluminio (mg. ${ }^{-1}$ ) & 0,2 & 0,20 & 0,05 to 0.2 & 0,10 \\
\hline Zinc (mg. $\mathrm{l}^{-1}$ ) & 3 & $\mathrm{NE}$ & 5 & 5 \\
\hline Hierro (mg.l $\left.\mathrm{l}^{-1}\right)$ & 0,30 & $\mathrm{NE}$ & 0,30 & 0,30 \\
\hline
\end{tabular}


Tabla 4. Valores guías o directrices para protección de la vida acuática y uso agrícola

\begin{tabular}{|c|c|c|c|c|c|c|c|}
\hline \multirow[t]{2}{*}{ Parámetro } & \multirow{2}{*}{ 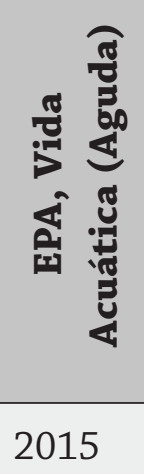 } & \multirow{2}{*}{ 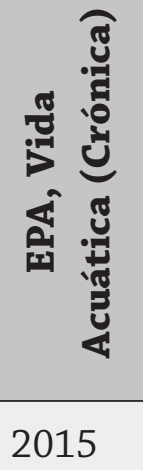 } & \multirow{2}{*}{ 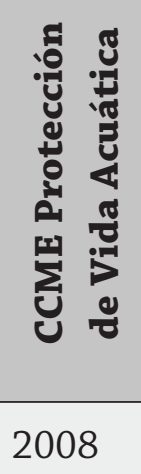 } & \multicolumn{2}{|c|}{$\begin{array}{l}\text { CCME Protección para } \\
\text { Uso Agrícola } 2008\end{array}$} & \multirow{2}{*}{ 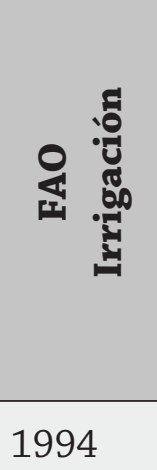 } & \multirow{2}{*}{ 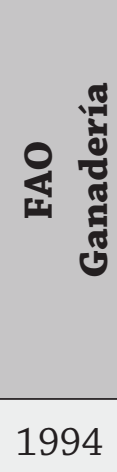 } \\
\hline & & & & Irrigación & Ganadería & & \\
\hline $\mathrm{pH}$ & $\mathrm{NE}$ & $6,5-8,5$ & $\mathrm{NE}$ & $\mathrm{NE}$ & $\mathrm{NE}$ & $6.5-8.4$ & $\mathrm{NE}$ \\
\hline TSS & 22,00 & 22,00 & 45,00 & & & & \\
\hline Aluminio (mg. $\left.\mathrm{l}^{-1}\right)$ & 0,75 & 0,087 & 0,005 & 5,00 & 5,00 & 5 & 5 \\
\hline Cobre (mg..$\left.^{-1}\right)$ & 0,0027 & 0,0021 & 0,002 & 1,00 & 1,00 & 0.05 & 0.5 \\
\hline Cromo (mg. $\left.\mathrm{l}^{-1}\right)$ & 0,139 & 0,0182 & 0,001 & 0,008 & 0,05 & 0.1 & 1 \\
\hline Hierro (mg. $\mathrm{l}^{-1}$ ) & 0,3 & 1 & 0.3 & 5,00 & NE & 5 & $\mathrm{NE}$ \\
\hline Manganeso(mg. $\left.1^{-1}\right)$ & $\mathrm{NE}$ & $\mathrm{NE}$ & $\mathrm{NE}$ & 0,20 & $\mathrm{NE}$ & 0.2 & 0.05 \\
\hline Talio (mg..$^{-1}$ ) & $\mathrm{NE}$ & $\mathrm{NE}$ & 0.0008 & $\mathrm{NE}$ & NE & $\mathrm{NE}$ & \\
\hline Zinc $\left(\mathrm{mg} . \mathrm{l}^{-1}\right)$ & 0,0274 & 0,0276 & 0,03 & 5,00 & 50,00 & 2 & 24 \\
\hline
\end{tabular}

\section{Estimación de la calidad del agua mediante el software CWQI}

Se calculó el índice de calidad de agua utilizando el software CWQI 1.0 desarrollado por la CCME que proporciona la manera más conveniente para resumir datos complejos y facilitar su interpretación. El CWQI incorpora tres elementos: a) Alcance, número de variables que no cumplen los objetivos de calidad del agua; b) Frecuencia, número de veces que no se cumplen estos objetivos; y c) Amplitud, cantidad por la que no se cumplen los objetivos. El índice produce un número entre 0 (agua de menor calidad) y 100 (agua de mejor calidad), por lo que la calidad del agua se puede clasificar en categorías: Excelente (95-100), Buena (80-94), Regular (65-79), Marginal (45-64) y pobre (0-44). El índice se puede usar tanto para monitorear cambios en un sitio a lo largo del tiempo como para realizar comparaciones entre sitios (CCME, 2001). El software de índice de calidad de agua aplica la siguiente ecuación: 


$$
C C M E W Q I=100-\left(\frac{\sqrt{F_{1}^{2}+F_{2}^{2}+F_{3}^{2}}}{1,732}\right)
$$

Se estimó la calidad del agua, introduciendo primeramente los objetivos de calidad, tomando en cuenta los valores máximos admisibles o recomendados por las normas a usar, luego se introdujeron los datos obtenidos y se corrió el programa, obteniendo valores numéricos para la calidad de las aguas de cada sitio muestreado. (CCME, 2014)

\section{RESULTADOS Y DISCUSIÓN}

\section{Aguas subterráneas}

En el sitio de Peñas Blancas, el pH se encontró ligeramente por debajo del rango establecido por la CAPRE, EPA y CCME para consumo humano ya que lo establecen en un rango muy estrecho. En el Túnel Azul, los valores encontrados para aluminio y zinc se encuentran dentro de los rangos establecidos por la CAPRE, OMS, USEPA y CCME y FAO para todos los usos del agua, no obstante, supera en el primer muestreo las directrices que establece la CCME para protección de la vida acuática. (Tabla 5)

Tabla 5. Parámetros de aguas subterráneas que incumplen al menos una normativa en los cinco muestreos realizados desde septiembre hasta diciembre del 2013

\begin{tabular}{|c|c|c|c|c|c|c|}
\hline Sitio & Parámetros & 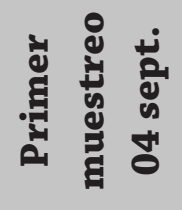 & 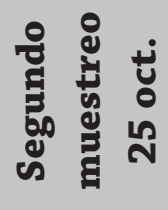 & 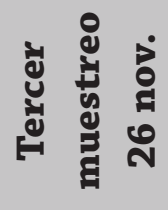 & 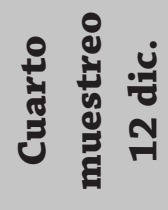 & 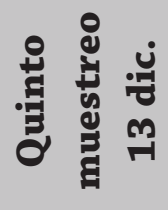 \\
\hline Peñas Blancas & $\mathrm{pH}$ & 6,815 & 6,46 & 6,58 & 6,71 & 6,73 \\
\hline \multirow{2}{*}{ Túnel Azul } & aluminio $\left(\mathrm{mg} \cdot \mathrm{l}^{-1}\right)$ & 0,011 & $<\mathrm{LD}$ & ND & $<\mathrm{LD}$ & $<\mathrm{LD}$ \\
\hline & zinc $\left(\mathrm{mg} . \mathrm{l}^{-1}\right)$ & 0,11 & 0,094 & 0,096 & 0,086 & 0,085 \\
\hline
\end{tabular}

\section{Aguas superficiales}

La tabla 6 muestra que en todos los sitos el aluminio total o disuelto se encontró en concentraciones superiores a lo que establecen las normas para la protección de la vida acuática, consumo humano y uso agrícola según la CCME, CAPRE, USEPA y FAO. El hierro total o disuelto superó los valores establecidos por la EPA y CCME para protección de la vida acuática y consumo humano según la CCME, CAPRE y USEPA, además sobrepasó los valores establecidos para el uso agrícola en el plantel La Estrella y El Salto según la CCME, no obstante, en el Sardinillo se 
encuentra ligeramente superior a los que establecen dichas normas, lo cual aún no llega a ser significativo; ya que las aguas de este sitio pueden considerarse con potencial para el uso de consumo humano.

Tabla 6. Parámetros de agua superficial que incumplen al menos una normativa en los cinco muestreos realizados desde septiembre hasta diciembre del 2013

\begin{tabular}{|c|c|c|c|c|c|c|}
\hline Sitio & Parámetros & 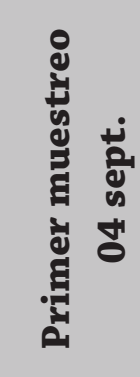 & 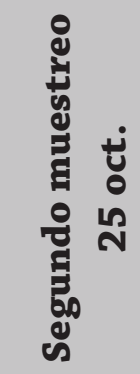 & 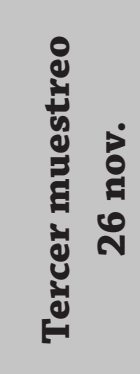 & 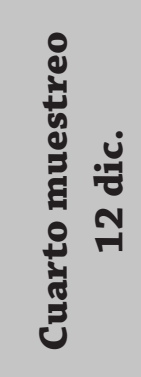 & 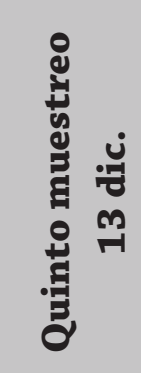 \\
\hline \multirow{4}{*}{ 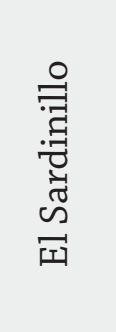 } & Aluminio Total (mg. $\left.\mathrm{l}^{-1}\right)$ & 0,359 & 0,06 & 0,784 & 0,642 & 3,4 \\
\hline & Aluminio disuelto (mg. $1^{-1}$ ) & 0,04 & 0,007 & 0,08 & 0,103 & 0,232 \\
\hline & Hierro Total (mg.l $\left.{ }^{-1}\right)$ & 0,594 & $<\mathrm{LD}$ & 0,801 & 0,715 & 4,201 \\
\hline & Manganeso (mg. ${ }^{-1}$ ) & 0,039 & $<\mathrm{LD}$ & $<\mathrm{LD}$ & $<\mathrm{LD}$ & 0,098 \\
\hline \multirow{7}{*}{ 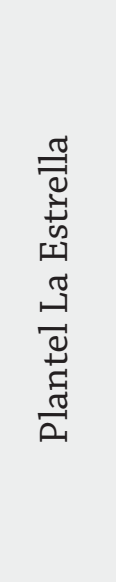 } & Aluminio Total (mg.l $\mathrm{l}^{-1}$ ) & 2,156 & 1,848 & 10,419 & 9,992 & 10,171 \\
\hline & Aluminio disuelto (mg. $1^{-1}$ ) & ND & 0,563 & $<\mathrm{LD}$ & 0,097 & 0,357 \\
\hline & Hierro Total (mg.l $\left.{ }^{-1}\right)$ & 4,601 & 3,698 & 23,757 & 21,312 & 20,737 \\
\hline & Hierro disuelto (mg. $\left.1^{-1}\right)$ & 0,136 & $<\mathrm{LD}$ & 0,295 & 0,132 & 0,378 \\
\hline & Manganeso Total (mg.l $\left.\mathrm{l}^{-1}\right)$ & 2,315 & 0,8 & 1,249 & 5,317 & 1,787 \\
\hline & Zinc $\left(\mathrm{mg} . \mathrm{l}^{-1}\right)$ & 0,03 & $<\mathrm{LD}$ & $<\mathrm{LD}$ & 0,045 & 0,052 \\
\hline & TSS (mg.l $\left.\mathrm{l}^{-1}\right)$ & 118,5 & & 425,4 & 591 & 337,7 \\
\hline
\end{tabular}




\begin{tabular}{|c|c|c|c|c|c|c|}
\hline Sitio & Parámetros & 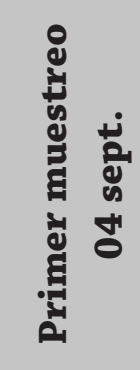 & 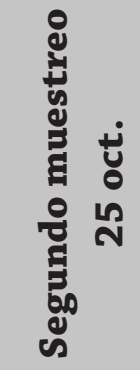 & 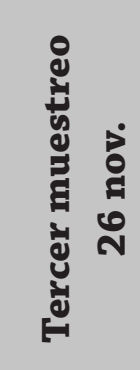 & 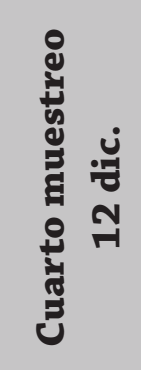 & 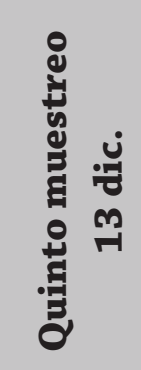 \\
\hline \multirow{11}{*}{ 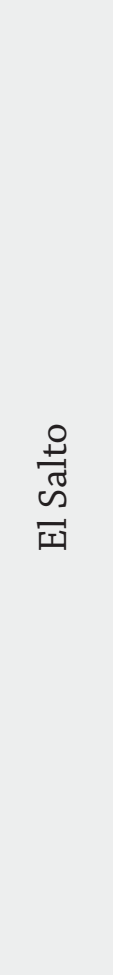 } & Aluminio Total (mg. $\mathrm{l}^{-1}$ ) & 13,691 & 1,934 & 11,791 & 3,306 & 8,31 \\
\hline & Aluminio disuelto $\left(\mathrm{mg} \cdot \mathrm{l}^{-1}\right)$ & 1,104 & 0,773 & 0,02 & 0,252 & 0,215 \\
\hline & Cobre $\left(\mathrm{mg} . \mathrm{l}^{-1}\right)$ & 0,004 & $<\mathrm{LD}$ & ND & $<\mathrm{LD}$ & $<\mathrm{LD}$ \\
\hline & $\operatorname{Cromo}\left(\mathrm{mg} . \mathrm{l}^{-1}\right)$ & 0,021 & ND & ND & $<\mathrm{LD}$ & $<\mathrm{LD}$ \\
\hline & Hierro Total (mg. $\left.\mathrm{l}^{-1}\right)$ & 21,907 & 3,715 & 20,465 & 29,099 & 18,439 \\
\hline & Hierro (mg. $\left.\mathrm{l}^{-1}\right)$ & 1,421 & 0,256 & 0,197 & 0,307 & 0,292 \\
\hline & Manganeso total (mg..$\left.^{-1}\right)$ & 2,517 & 0,559 & 2,71 & 11,829 & 7,168 \\
\hline & $\begin{array}{l}\text { Manganeso disuelto } \\
\left(\mathrm{mg} \cdot \mathrm{l}^{-1}\right)\end{array}$ & 0,198 & 0,29 & 0,468 & 0,428 & 0,415 \\
\hline & Talio (mg..$^{-1}$ ) & ND & ND & $<\mathrm{LD}$ & 0,015 & ND \\
\hline & Zinc (mg. $\left.\mathrm{l}^{-1}\right)$ & 0,074 & $<\mathrm{LD}$ & $<\mathrm{LD}$ & 0,057 & 0,058 \\
\hline & TSS (mg. $\left.\mathrm{l}^{-1}\right)$ & 509 & 66,57 & 314,78 & 357 & 371 \\
\hline
\end{tabular}

En el plantel la Estrella y el Salto los sólidos suspendidos totales (SST) superaron los valores establecidos por la EPA y CCME para la protección de la vida acuática, esto probablemente es debido a que la mayoría de las minerías artesanales descartan sus aguas residuales al río sin ningún tratamiento, las cuales contienen grandes cantidades de SST provocando alteración en las concentraciones de metales como aluminio y hierro, lo que podría ocasionar que muchas especies acuáticas se extingan entre ellos los peces, ya que éstos metales se acumulan en ciertos órganos vitales ocasionándoles la muerte. (Slaninova A., Machova J., Svobodova Z., 2014).

\section{Índice de calidad de agua para sitios de agua subterránea}

Para el sitio Peñas Blancas, la calidad del agua resultó ser excelente para todos los usos: consumo humano, irrigación y ganadería, considerados en las normas EPA, CCME, CAPRE, FAO y OMS. Sin embargo, para consumo humano según EPA y CCME el índice no alcanzó la valoración máxima de 100 debido a que norman el pH en un rango más estrecho $(6,5-8,5)$ que en uno de los 
muestreos el valor fue ligeramente inferior $(6,46)$, lo cual no llega a ser tan importante. (Figura 1).

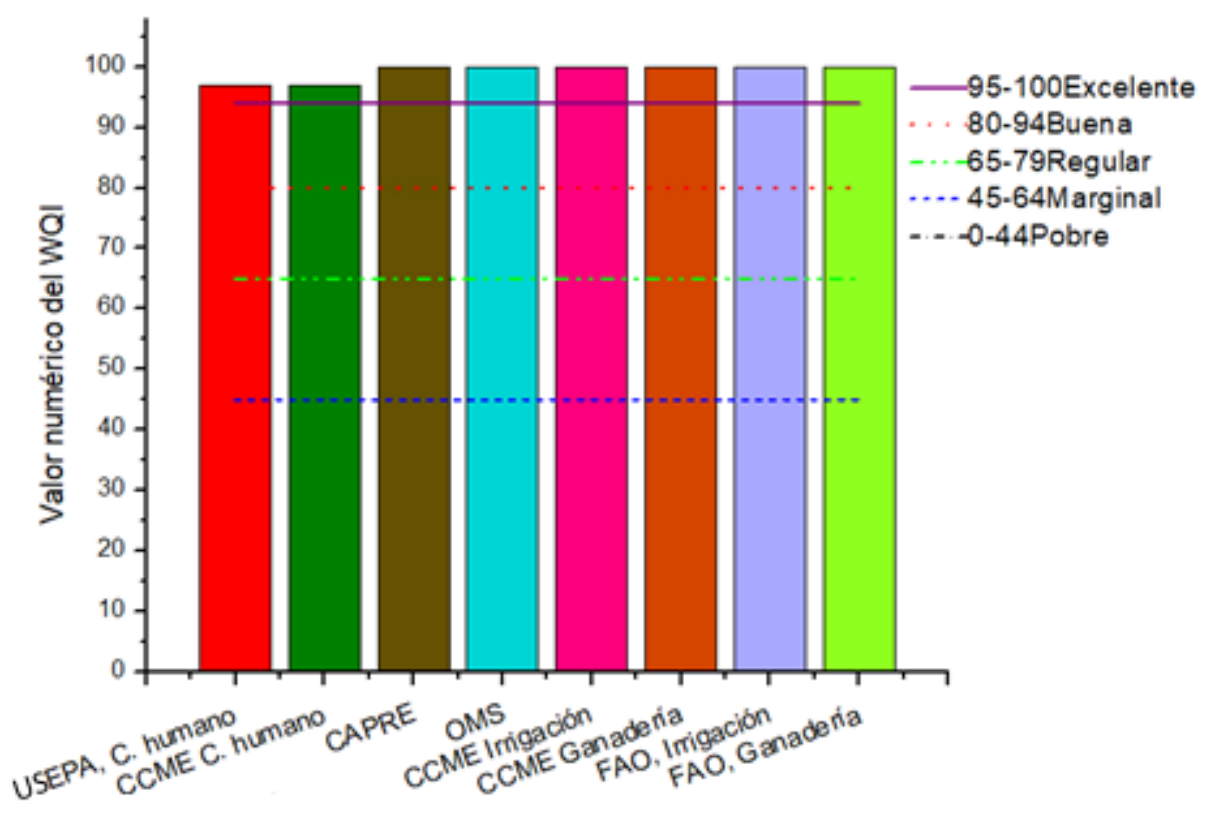

Figura 1. Índice de Calidad del agua de Peñas Blancas

En el Túnel Azul, el índice de calidad de agua, al igual que peñas Blancas, resultó ser excelente para todos los usos, para consumo humano (considerado en las normas EPA, CAPRE, OMS) e irrigación y ganadería según las directrices (considerado en CCME y FAO). (Figura 2). 


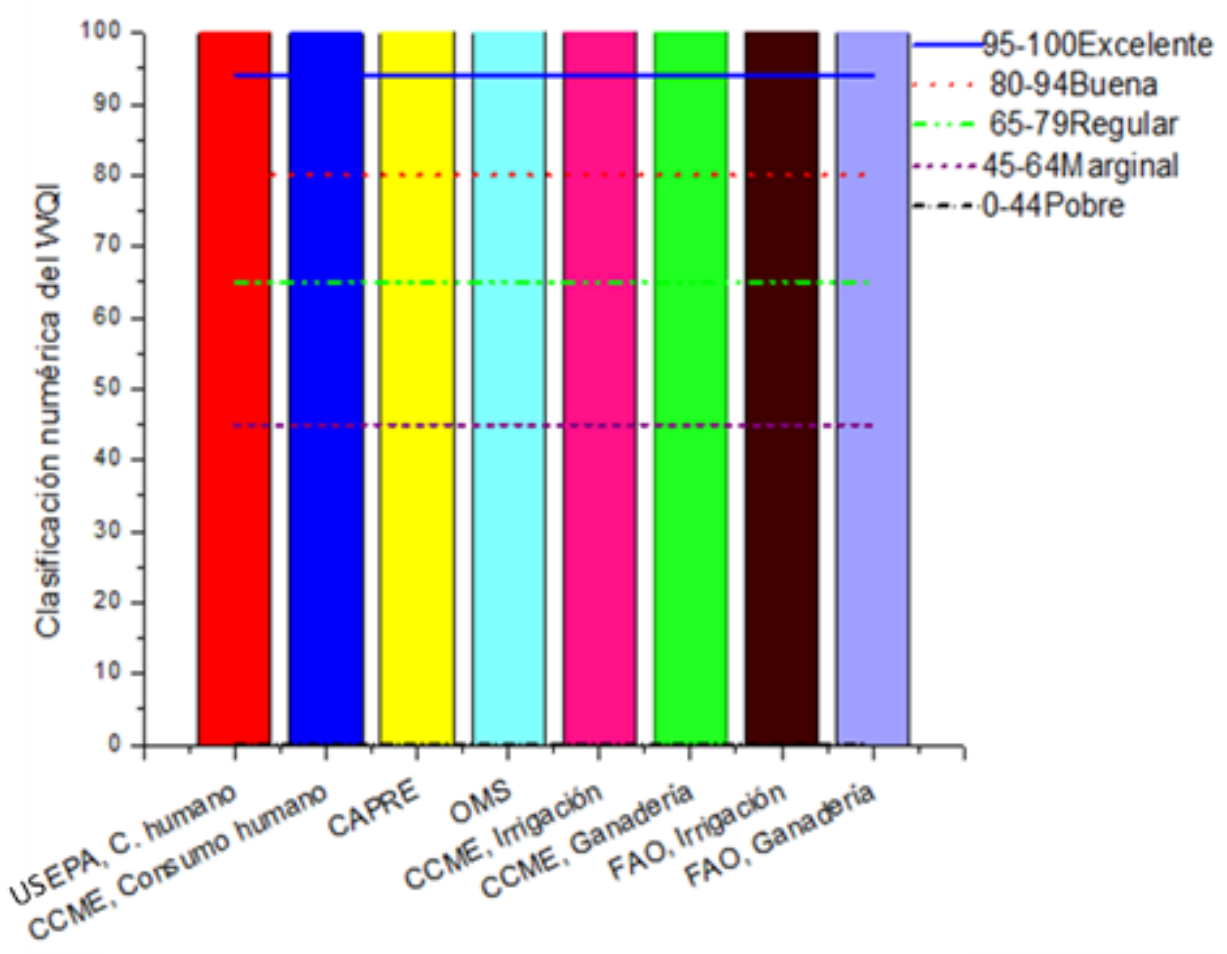

Figura 2. Índice de calidad de agua del Túnel Azul

En los dos sitios evaluados, la calidad de las aguas es excelentes para todos los usos, sin embargo, comparando los dos sitios y con base en lo que establece la norma EPA y CCME para consumo humano, las aguas del Túnel Azul resultaron tener mejor índice de calidad que las de Peñas Blancas.

\section{Índice de calidad de aguas superficiales}

El Sardinillo, es un sitio de referencia localizado aguas arriba donde no hay influencia de la actividad minera. (Figura 3). El índice de la calidad de agua resultó ser regular a buena para consumo humano según la directriz canadiense CCME, OMS y USEPA por lo que se considera que podría ser viable para este uso; para la protección a la vida acuática, según la CCME y USEPA, tiene un índice de calidad que va de pobre a buena, sin embargo, resultó ser un agua excelente para el uso agrícola según las normativas CCME y FAO. 


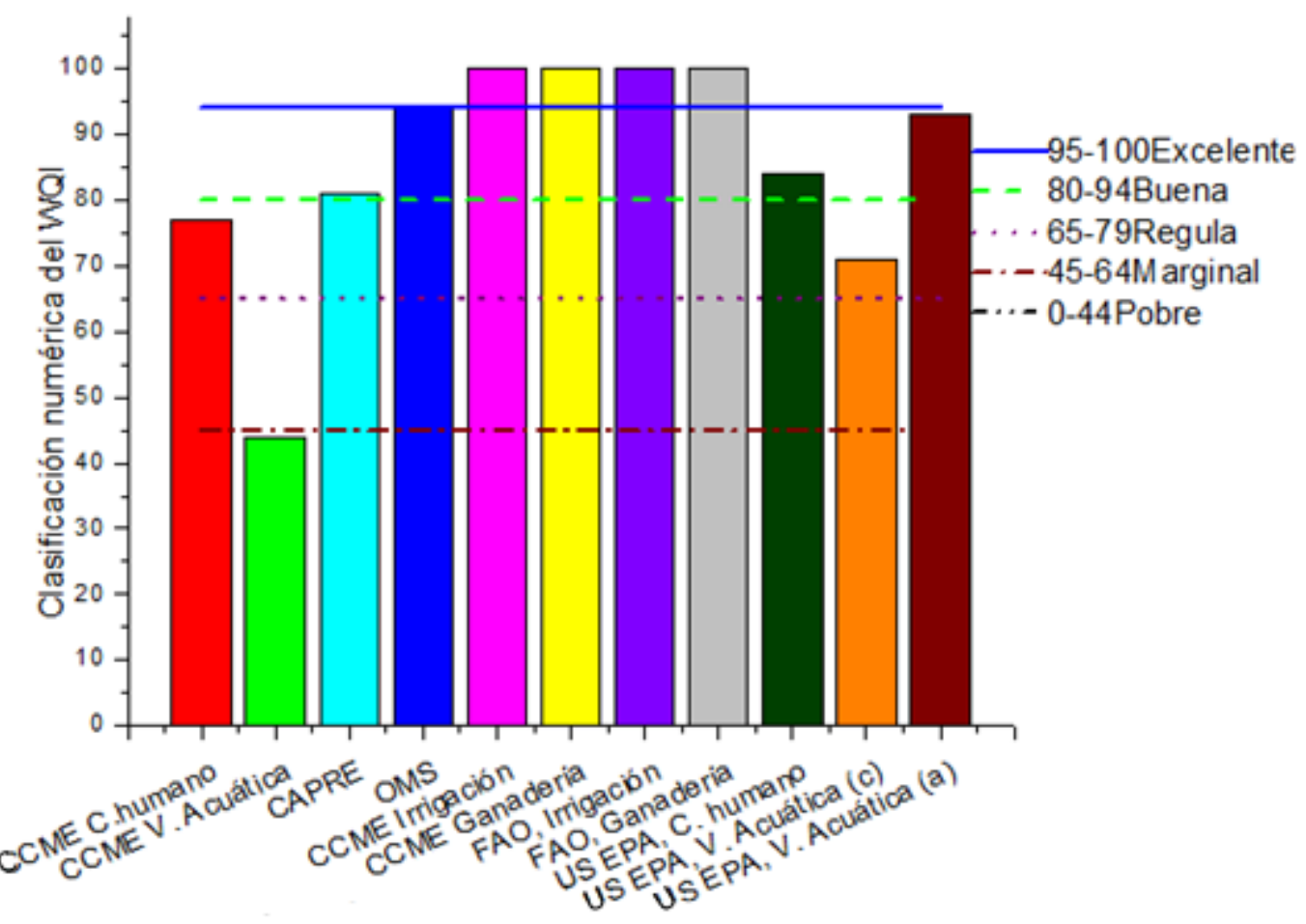

Figura 3. Índice de calidad de agua del Sardinillo

Al igual que en el caso anterior, se calculó el índice de calidad de agua para las aguas del plantel La Estrella, el cual está ubicado próximo, río abajo, donde el botadero del mismo nombre vierte sus efluentes. Los índices se muestran en la figura 4.

El índice de calidad de agua del plantel La Estrella resultó ser pobre a marginal para consumo humano según las directrices CCME, CAPRE, OMS y EPA; para la protección de la vida acuática, pobre a marginal según la CCME y EPA (aguda y crónica) lo que indica que muchas especies acuáticas no podrían sobrevivir en las aguas de este sitio debido a su mala calidad, no obstante, el agua para el uso agrícola resultó ser regular según CCME y excelente considerando los valores que establece la FAO para irrigación y ganadería. 


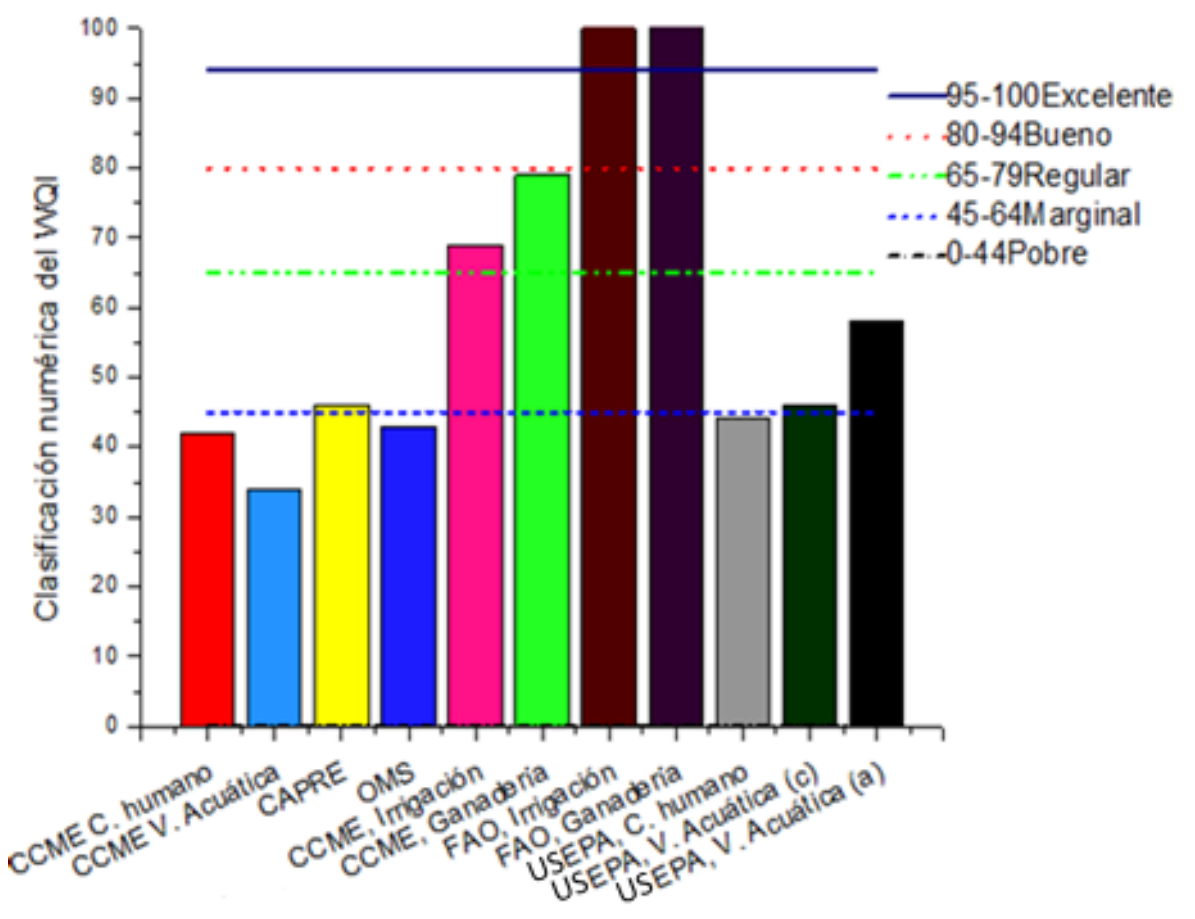

Figura 4. Índice de calidad de agua del plantel La Estrella

El tercer sitio de importancia para el análisis de las aguas superficiales es El Salto, el cual está ubicado aguas abajo. La calidad del agua de este sitio resultó ser pobre para consumo humano y protección de la vida acuática, sin embargo, según las consideraciones de CCME y EPA, esta agua resultó ser marginal a buena para irrigación y ganadería según la CCME y FAO. (Figura 5). 


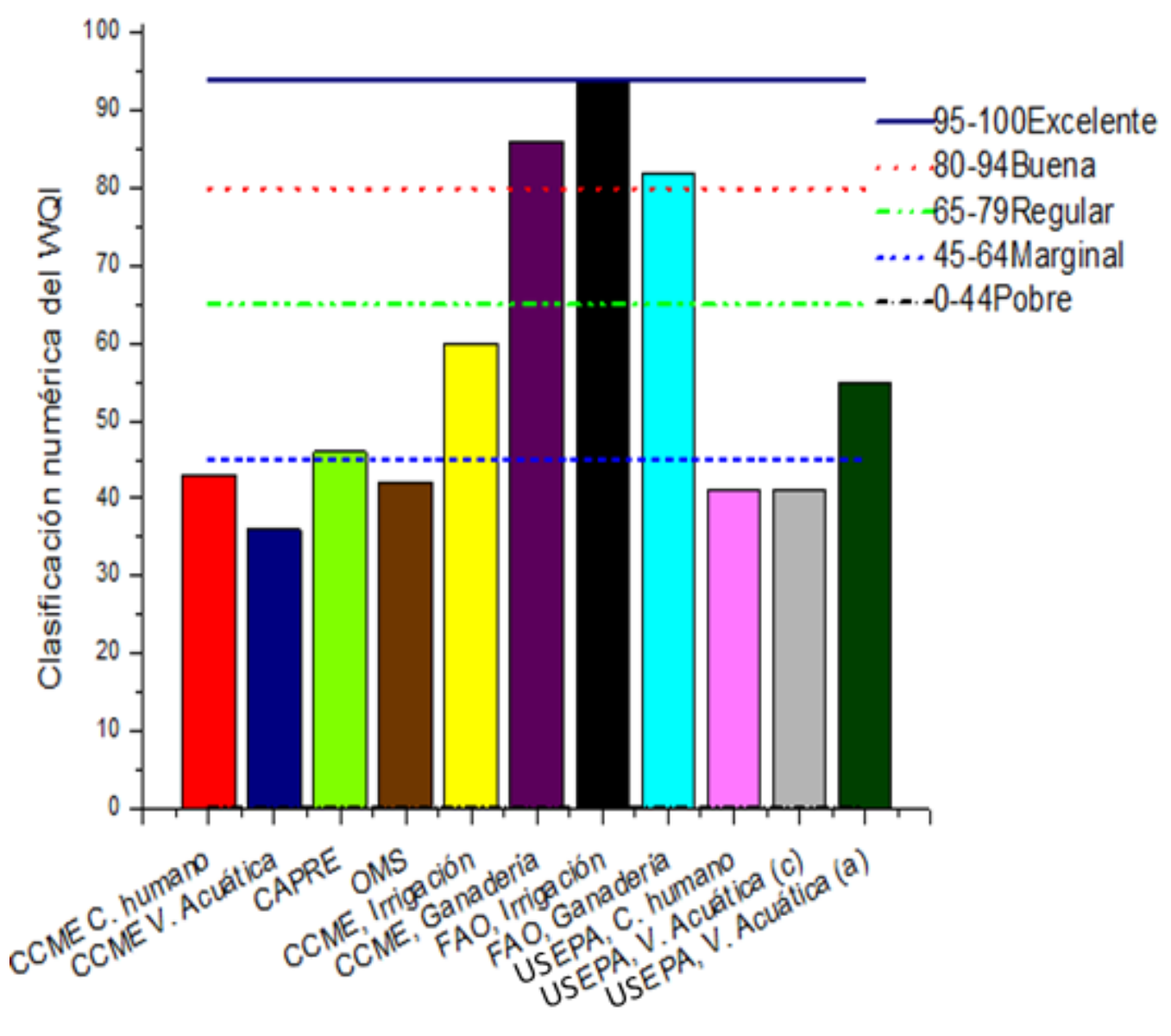

Figura 5. Índice de calidad de agua del Salto

Al realizar el cálculo del índice de calidad de agua en los tres sitios muestreados, se encontró que estas aguas pueden ser utilizadas para irrigación y ganadería, no así para consumo humano, sin embargo, considerando los parámetros analizados, El Sardinillo puede ser considerado con cierta viabilidad para el consumo humano, así como para albergar vida acuática, no así en El Salto y plantel La Estrella. El principal problema del plantel La Estrella y El Salto son los sólidos suspendidos totales, ya que éstos tienen un impacto elevado en la calidad del agua y en el ecosistema acuático. El sedimento suspendido en el agua puede irritar las branquias de los peces ya que son acumulados en este órgano (Susfalk, R., Fitzgerald, B., Knust, A. \& Desert Research Institute, 2008), además cuando estos sólidos se sedimentan, pueden sofocar organismos que habitan en el fondo del río y cubrir el lecho grueso que ciertos peces requieren para desovar (Sullivan, J. D., U.S.Department of the Interior \& U.S.Geological Survey, 2000). No obstante, no se puede descartar el riesgo microbiológico; puesto que este último parámetro no fue analizado. 


\section{CONCLUSIONES}

- Los índices de calidad de las aguas subterráneas Peñas Blancas y Túnel Azul indican que estas aguas son aptas para consumo humano, irrigación y ganadería.

- El Sardinillo presenta un índice de calidad del agua regular a buena para consumo humano según CCME, CAPRE y USEPA, por lo que se considera con potencial para este uso; además con índice de calidad que va desde pobre a buena para la protección de la vida acuática y excelente para irrigación y ganadería.

- El plantel La Estrella y El Salto presentan similitud en la calidad de las aguas para consumo humano y protección de la vida acuática con índices de calidad que oscilan entre pobre y marginal según CCME, CAPRE y USEPA. Para el uso agrícola El plantel La Estrella presentó una calidad de agua desde regular a excelente y El Salto desde marginal a buena según las consideraciones de CCME y FAO. Lo que indica que estas aguas no son adecuadas para el consumo humano, ni para albergar vida acuática, no obstante, podrían tener potencial para el uso agrícola. Los parámetros microbiológicos no fueron analizados lo que también podría influir en la calidad del agua en estos sitios estudiados.

\section{AGRADECIMIENTOS}

- A la Agencia Austriaca para la Cooperación Internacional en Educación e Investigación (OEAD), dentro del marco de APPEAR, y en unión con la Universidad de Vienna (UNIVIE) de Austria, la Universidad Nacional Santiago Antúnez de Mayolo (UNASAM), Perú, a través del proyecto de Biorremediación de Sitios Contaminados: Investigación y Educación (BIOREM); por brindar los medios económicos para la realización de este estudio de investigación.

- Dra. Katia Montenegro Rayo por su valioso aporte técnico en la ejecución de esta investigación.

- Ing. Francisco Ramón Vallecillo Galeano por su colaboración durante la investigación.

- A la Alcaldía de Santo Domingo, Chontales por su colaboración en la realización del monitoreo de las fuentes de agua subterráneas y superficiales. 


\section{REFERENCIAS}

APHA, AWWA, WEF Standard Methods for the Examination of Water and. (2012). Standard Methods for the Examination of Water and wastewater 22nd edition. Washington: American Public Health Association.

Ayers R. S., Westcot D. W., \& FAO. (1985). Water quality for agriculture. California: Food and agriculture Organization of the Unites Nations.

CAPRE. (1994). Normas de Calidad del Agua para Consumo Humano. Obtenido de Normas de calidad del agua para consumo humano.

CCME. (2001). CCME Water Quality Index 1.0 User's Manual.

CCME. (2014). Canadian Water Quality Guidelines for the Protection of Aquatic Life. Summary Table.

CCME. (28 de Junio de 2019). Guidelines for Canadian Drinking Water Quality - Summary Table. https://www. canada.ca/en/health-canada/services/ environmental-workplace-health/ reports-publications/water-quality/ guidelines-canadian-drinking-waterquality-summary-table.html

Espinoza, T., \& Espinoza Benavides L. (2005). Impacto de la mnería en la calidad en la microcuenca del Río Artiguas s. Énfasis en metales pesados. Managua.
Flores, M., E. (2009). Conflictos socioecológicos Minería Ficha N $^{\circ} 28$. Fuhem.

Nolasco, S., CEICOM. (2011). Impactos de la Minería Metálica en Centroamérica. https://rb.gy/cukhix

OMS. (2006). Guías para la calidad del agua potable. Primer apéndice a la tercera edición. Vol 1. . Primer apéndice a la tercera edición. Vol 1. Recomendaciones. Tercera edición.

OMS. (14 de Junio de 2019). Agua. https:// www.who.int/es/news-room/factsheets/detail/drinking-water

Picado, F., Cuadra, S., Mendeoza, A.,\& Barmen, G. (2010). Ecological, Groundwater, and Human Health Risk Assessment in a Mining Region of Nicaragua. Risk Analysis, 916-933.

Slaninova A., Machova J., Svobodova Z. (2014). Fish kill caused by aluminium and iron contamination. En U. U. Agriculture, Integrated Investigations of Environmental Effects of Historical Mining in the Animas River Watershed, San Juan County, Colorado (págs. 573581).

Sullivan, J. D., U.S.Department of the Interior \& U.S.Geological Survey. (2000). Nutrients and Suspended Solids in Surface Waters of the Upper Illinois River Basin in Illinois, Indiana, 
and Wisconsin,1978-97. Middleton, Wisconsin.

Susfalk, R., Fitzgerald, B., Knust, A. \& Desert Research Institute. (2008). Characterization of Turbidity and Total Suspended Solids in the Upper Carson River, Nevada. DHS Publication No. 41242.

USEPA. (20 de Julio de 2017). National Recommended Water Quality Criteria Aquatic Life Criteria Table.

WHO. (2011). Guidelines for drinking-water quality. 4 th ed. 